\title{
New approach needed for diagnosis of human brucellosis in Morocco
}

\author{
Aicha Qasmaoui ${ }^{1.2}$, Bouchra Belkadi ${ }^{2}$, Farida Ohmani $^{1}$, Karima Halout1, Reda Charof $^{1}$ and Jamila Hamamouchi ${ }^{1}$ \\ ${ }^{1}$ Laboratory of Epidemic Diseases, Department of Medical Bacteriology, National Institute of Hygiene, Avenue Ibn Batouta, \\ Rabat, Morocco. \\ 2 Team of Microbiology and Molecular Biology, Research Center for Plant and Microbial Biotechnology, Biodiversity and \\ Environment, Faculty of Sciences, Mohammed V University of Rabat, Morocco
}

\begin{abstract}
Human brucellosis is an infectious disease, caused by different species of the genus Brucella. It is a mandatory notifiable disease in most countries, including Morocco, and thus requires special vigilance on the part of health agencies. Prevention of this disease is based on surveillance and prevention of risk factors. Its management is based on serological or other diagnostic tests. The National Laboratory of Epidemic Diseases received 17 blood samples of human origin for notified cases of brucellosis in a region of southern Morocco. Biological confirmation of these samples was performed by Rose Bengal test and ELISA IgM test. The results showed a positivity rate of $88 \%$ proving the important role of serological tests in the diagnosis of brucellosis. Other rapid and efficient techniques are becoming essential for the confirmation of cases, taking into account the advanced biosafety procedures for these highly infectious pathogens.
\end{abstract}

\section{Introduction}

Recognized as a re-emerging neglected disease, brucellosis is a zoonosis that affects humans and various domestic and wild animal species and remains a health problem in developing countries. [1,2]. It is transmitted directly or indirectly to humans through contact with contaminated animals or their products [3], they have been classified as one of the most important neglected professional risks worldwide by the Office International des Epizooties (OIE), the World Health Organization (WHO) and the Food and Agriculture Organization of the United Nations (FAO)[4,5]. Professional exposure and consumption of raw milk is one of the major sources of infection [6,7]. This disease is caused by gram-negative bacteria of the genus Brucella, which currently includes 12 species, of which only four are particularly pathogenic for humans: Brucella abortus, Brucella canis, Brucella suis and Brucella melitensis [8,9].

It is a widespread disease in the world and one of the most important zoonoses worldwide in many countries, with 500 thousand cases reported each year according to WHO [10]. This pathology remains as endemic in many countries [11]. In Africa little data is available on human brucellosis [12]. In Morocco, it is part of the list of notifiable diseases. Indeed, any suspected, probable or confirmed case must be immediately reported to the delegations of the Ministry of Health, which will forward the information to the Directorate of Epidemiology and the Fight against Diseases. An epidemiological investigation is initiated and samples are sent to the national reference laboratory for epidemic diseases at the National Institute of hygiene. [13,14].

In humans, brucellosis can cause a multitude of clinical symptoms such as mild influenza-like illness, chronic fatigue syndrome, acute febrile illness and spontaneous abortion. Internal organ complications such as meningitis, endocarditis, encephalitis, arthritis, orchitis, prostatitis, and spondylitis have also been reported. The disease can last for years, and treatment requires patient adherence to costly and prolonged therapy $[3,6]$.With non-specific signs and symptoms the diagnosis of brucellosis is difficult to detect, which requires laboratory confirmation according to WHO recommendations $[8,12,15]$.

The definitive diagnosis of brucellosis is based on the culture of Brucella, this is the reference technique but the bacteria grows relatively slowly and a successful culture in the laboratory can be difficult due to the intracellular nature of the bacteria $[16,17]$. Serological techniques are the most widely used; these are different techniques for testing antibodies, including the WHO reference method of Wright's serum agglutination, the Rose Bengal method, the complement fixation method and the enzyme-linked immunosorbent assay (ELISA)[18]. They are inexpensive and less time consuming to perform and are easy to interpret [5]. Currently PCR facilitates a rapid, sensitive and specific detection and also allows to define species and biovar by amplification of specific regions [19].

No vaccine is used in humans, so the severity of this disease and the lack of vaccines suitable for humans have led to the classification of Brucella as a bioterrorism agent [20, 21, 22].

Prevention methods include health education to reduce occupational and dietary risks [23]. Control activities must be coordinated and shared between the public health and animal health sectors [24], therefore the eradication of this disease depends largely on its elimination in animals [25]. Due to the importance of brucellosis surveillance in the public health system and knowing the crucial role of the

\footnotetext{
*Corresponding author: qasmaouiaicha21@gmail.com
} 
laboratory in this control and prevention program, the present work aims to document cases of brucellosis reported during the month of June 2017 during an epidemic that struck during this year a region of southern Morocco. The serological diagnosis for the confirmation of cases and recommendations for a better detection of the infectious agent will also be presented.

\section{Material and method}

17 blood samples from patients who had been seen by a private or public doctor. After examination, they were considered suspected of having symptoms of brucellosis. The blood samples were sent to the laboratory of epidemic diseases of the National Institute of Hygiene in Rabat for diagnosis and confirmation. Blood samples were centrifuged at $1500 \mathrm{rpm}$ for 10 minutes. The collected sera were examined for Brucella antibodies using the Rose Bangle test and ELISA.

\subsection{Rose Bengale test}

The Rose Bengale test is a qualitative slide agglutination test for the detection of anti-Brucella antibodies. It allows the serological diagnosis of brucellosis due to Brucella melitensis, Brucella abortus, Brucella bovis or Brucella suis by $\operatorname{IgG}$ detection (BioRad ${ }^{\circledR}$, France). Sera were considered positive when agglutination was visible.

\subsection{ELISA IgM Test:}

The use of enzyme-linked immunosorbent assay (ELISA) for the detection of IgM antibodies against Brucella species was performed by the Kit (ELISA IGM DRG, German) following the manufacturer's instructions. Only samples above $11 \mathrm{DU} / \mathrm{ml}$ were considered positive. The sensitivity and specificity provided by the manufacturer were $100 \%$.

Samples were analyzed according to good laboratory practices to limit the risk of contamination by these highly pathogenic agents.

\section{Result}

17 sera processed at the Epidemic Diseases Laboratory as part of a brucellosis suspension for confirmation. $88,2 \%$ of the cases $(15 / 17)$ were positive in both serological tests: Rose Bengal antigen reaction and $\operatorname{IgM}$ immunological detection by ELISA. 2 negative cases represent $11.8 \%$, one of the two negative cases was a control case who lived in the same area affected by the epidemic for more than a year and had none of the clinical signs of brucellosis. The other suspect case was undiagnosed but had clinical symptoms and was also negative for both tests performed.
The average age of the 15 patients diagnosed as positive was 43 years. 22 to 67 years of age, of which $60 \%$ were men and $40 \%$ were women. All patients were found to have been infected through the consumption of raw milk or undercooked liver. These confirmed cases are of urban origin according to the interrogation conducted.

\section{Discussion}

Human brucellosis is a zoonotic disease, classified by the WHO as one of the major neglected diseases of the world and poorly known by physicians [26]. It is endemic in Africa including Morocco [27] Due to the non-specificity of the clinical signs, which makes it difficult to diagnose by confusing it with other infectious or non-infectious diseases and therefore generates problems for a rapid diagnosis[28,29]. Therefore, their biological diagnosis in the laboratory is essential for a confirmation of a suspected case to a confirmed case [30].

In front of the difficulty of a diagnosis by culture which is long with false negatives and a great risk for the laboratory staff $[8,15]$. Serodiagnosis remains an accessible and inexpensive method for the detection of brucellosis whose sensitivity and specificity vary according to the studies [18]. We can adopt it as a first-line method for the diagnosis of brucellosis and to set up a treatment [12].

We reported here notified cases of brucellosis in 2017 where Morocco experienced an epidemic in the province of Laayoune. Several cases of brucellosis were reported that have clinical symptoms this disease, as part of its investigation, the laboratory of Epidemic Diseases treated 17 suspect samples. 15 cases were found to be positive by Rose Bengal test and IgM ELISA test. The first is used for screening and must be confirmed by another test which is recommended by many international organizations for the diagnosis of human brucellosis. [31, 32]. The ELISA is a confirmatory test, simple and easy to perform and interpreted with less time can be used combined with the first test for an easy diagnosis of the disease. This advantage gives it priority of use in routine as a diagnostic technique. Anti-Brucella IgM antibodies are indicative of acute infection. Some studies give a specificity of $100 \%$ for IgM detection by ELISA for the diagnosis of acute brucellosis.but the definitive diagnosis of brucellosis should not be based solely on the detection of anti-Brucella IgM antibodies and should be evaluated in the context of the clinical history and epidemiological background [18]

However, the antibodies directed against the smooth polysaccharides of the bacterium persist for a long time after recovery, thus raising the problem of the positivity of cases in endemic areas. [10]. Also IgM 
antibodies may be detected due to cross-reaction with other clinical conditions. Confirmation by the real-time PCR technique for its sensitivity and specificity is therefore necessary. $[19,33]$. We also observed one case with clinical signs but was diagnosed negative. This highlights the need to perform other tests such as IgG ELISA and PCR taking into account the cost, time and risk of handling. This finding is in agreement with the results of other studies that have shown the combined specificity of both IgG and IgM tests can go up to $100 \% \cdot[26,30,33]$. Also, in our study, the presence of a control case in our study could overestimate the specificity of the test which requires its interpretation in a clinical context [8].

Cases are present in all age groups and overall, both sexes were equally affected, demographics did not show a difference between the two sexes. We find $40 \%$ of females and $60 \%$ of males diagnosed positive for Brucella. The mean age of the patients was $43 \pm 12$ years. Our results seem to be consistent with other investigations [34].

We found that all diagnosed cases are of urban origin and the consumption of raw milk and undercooked liver are the risk factors and sources of contamination. This has also been reported in several studies $[6,24]$ HIV-positive cases were treated with a six-week regimen of a combination of doxycycline and other drugs, six weeks of a combination of doxycycline and rifampicin, according to the treatment protocol recommended by the (WHO). Treatment of this condition is based on active antibiotics with good tissue and intracellular distribution [27] and any delay in treatment would be a factor in relapse upon treatment failure [12].

In humans, consumption of contaminated food and occupational contact are the main risks of infection [35]. In our study, the consumption of unpasteurized dairy products and poorly cooked livers appeared to be the only risk factor for this population, which is of urban origin, and which is accustomed to consuming raw milk and its derivatives, as documented by several studies [6, 35]. The human contamination is then linked to the animal infection especially in the southern regions which are the main affected areas where the seroprevalence rate of animal brucellosis is $2.8 \%$ to $3.3 \%$. [27, 36]. This region and among the administrative regions, $\mathrm{a}$ relatively high incidence was recorded for the study. Therefore, the best way to prevent brucellosis infection is to avoid eating undercooked meat and unpasteurized dairy products, including milk, cheese and ice cream. Pasteurization of dairy products for human consumption is important to prevent disease.
Moreover, vaccines for use in humans are not yet available, so the eradication and control of human brucellosis depends on the eradication of Brucella species from cattle, goats, pigs and other animals [37] However, vaccination of animals is one of the most effective methods to prevent and control this disease $[10,25]$. Therefore, the methods of control and prevention of this disease in humans depend heavily on the accuracy of diagnostic tools and on the use of diagnostic methods that are robust, reliable, sensitive, specific, and rapid. And the implementation of effective and safe vaccination programs and improved hygienic level and require sustainable collaborations for a single health, collaboration between the animal and human sectors with the sensitization of the population $[4,25]$

\section{Limits}

So assuming that there is an under-reporting of cases that remained undiagnosed which results in an underestimation of reported cases, Also in this study is that the laboratory received only one sample of each serum while the diagnosis of brucellosis requires two serum samples spaced 2 to 4 weeks apart to verify the increase in antibody levels, since the tests may be negative in the early stages of the disease $[5,10]$, It is therefore necessary to establish a close collaboration between the laboratory and the clinician for a good diagnosis. The addition of other diagnostic and confirmatory tests is essential. In this axis, our laboratory is currently invested to adopt the real-time PCR as a routine technique for the surveillance of brucellosis.

\section{Conclusion}

Brucellosis is an emerging infectious disease that poses a threat to all people in endemic areas such as Morocco, especially when the population is used to consuming raw dairy products. Due to non-specificity of clinical signs, biological diagnosis in the laboratory is essential for confirmation; Serodiagnosis is an accessible and inexpensive method in the detection of brucellosis whose sensitivity and specificity vary.Faced with this situation, there is an obligation to adopt a new approach to the diagnosis of this pathology in routine, the real-time PCR technique and combine it with serological tests for more sensitivity and specificity, while handling with less risk. This molecular diagnostic tool targets the Brucella genus and the differentiation between the major species, highly pathogenic for humans.

\section{References}

1. M.K .Ghanbari, H.A .Gorji, M. Behzadifar, N .Sanee, N. Mehedi, N.L. Bragazzi, One health approach to tackle brucellosis: a systematic review. Trop Med Health ;48:86.(2020) 
2. O . Madzingira, O.F. Fasina, C . Kalinda, H .Van Heerden, Seroprevalence of Brucellosis among Clinically Suspected Human Cases Presenting at Health Facilities in Namibia from 2012 to 2017. Biomed Env Sci ;Volume 34,: 232-235(2021)

3. K.J. Ryan, C.G .Ray, J.C. Sherris, editors. Sherris medical microbiology: an introduction to infectious diseases. 4th ed. New York: McGraw-Hill; (2004)

4. Organization $\mathrm{WH}$. $\mathrm{WHO}$ recommended strategies for the prevention and control of communicable diseases (2001)

5. P. de Figueiredo, T.A. Ficht, A. Rice-Ficht, C.A. Rossetti, L.G. Adams, Pathogenesis and Immunobiology of Brucellosis. Am J Pathol ;185:1505-17(2015)

6. R. Al Jindan, Scenario of pathogenesis and socioeconomic burden of human brucellosis in Saudi Arabia. Saudi J Biol Sci ;28:272(2021)

7. H. Battikh, A. Berriche, R. Zayoud, L. Ammari, R. Abdelmalek, B. Kilani, et al, Clinical and laboratory features of brucellosis in a university hospital in Tunisia. Infect Dis Now (2021).

8. P.Yagupsky, P . Morata, J.D. Colmenero, Laboratory Diagnosis of Human Brucellosis. Clin Microbiol Rev;33:e00073-19,(2020)

9. K.A. Franc ,R.C. Krecek, B.N. Häsler, ArenasA.M Gamboa, Brucellosis remains a neglected disease in the developing world: a call for interdisciplinary action. BMC Public Health ;18:125,(20218)

10. R. Shakir, Brucellosis. J Neurol Sci;420:117280,h (2021)

11. Y .Ben Lahlou, E. Benaissa, A. Maleb, M. Chadli, M. Elouennass. Pancytopenia revealing acute brucellosis. IDCases ;23:e01037,(2021)

12. K. Govindasamy. Human brucellosis in South Africa : a review for medical practitioners. $S$ Afr Med J;110:6,(2020)

13. A . Rguig, M. Ziani, A. Barkia, A. Benmamoune, O. El Menzhi Mise au Point: La surveillance épidémiologique au Maroc. Ministère Sante ;Junin 212:18,(2012)

14. Institut National d'Hygiène /Ministère de la sante. Plan_Strategique2017-2021.pdf. n.d.

15. R . Díaz, A .Casanova, J . Ariza, I . Moriyón, The Rose Bengal Test in Human Brucellosis: A Neglected Test for the Diagnosis of a Neglected Disease. PLoS Negl Trop Dis ;5:e950,(2011)

16. M.R. Hasanjani Roushan, S. Ebrahimpour, Human brucellosis: An overview. Casp J Intern Med ;6:46-7(2015)

17. M.Shakespeare. Zoonoses. 2nd ed. London; Chicago: Pharmaceutical Press; (2009)
18. J. Solís García del Pozo, S. Lorente Ortuño, E . Navarro, J. Solera, Detection of IgM Antibrucella Antibody in the Absence of IgGs: A Challenge for the Clinical Interpretation of Brucella Serology. PLoS Negl Trop Dis ;8:e3390,(2014)

19. A. Etemadi, R. Moniri, H. Neubauer, Y. Dasteh Goli, S. Alamian, Laboratory Diagnostic Procedures for Human Brucellosis: An Overview of Existing Approaches. Jundishapur J Microbiol ;12,(2019)

20. F.H. Kayser, Medical microbiology. Stuttgart; New York, NY: Georg Thieme Verlag; (2005)

21. P.M. Parker, J.N. Parker, Brucellosis: a medical dictionary, bibliography, and annotated research guide to Internet references. San Diego, CA: ICON Health Publications; (2004)

22. J. Enkelmann, K. Stark, M. Faber, Epidemiological trends of notified human brucellosis in Germany, 2006-2018. Int J Infect Dis ;93:353-8(2020)

23. Organization $\mathrm{WH}, W H O$ recommended strategies for the prevention and control of communicable diseases (2001)

24. S.A.A. detunji, G. Ramirez, A.R. Ficht, L. Perez, M.J. Foster, A.M. Arenas-Gamboa, Building the Evidence Base for the Prevention of Raw Milk-Acquired Brucellosis: A Systematic Review. Front Public Health ;8,(2020)

25. D. O'Callaghan, Human brucellosis: recent advances and future challenges. Infect Dis Poverty ;9:101,(2020)

26. R.A. Jindan, N. Saleem, A. Shafi, S.M. Amjad, Clinical Interpretation of Detection of IgM Anti-Brucella Antibody in the Absence of IgG and Vice Versa; a Diagnostic Challenge for Clinicians. Pol J Microbiol (2019);68:17,(2019)

27. A. Lemnouer, M. Frikh, A. Maleb, A. Ahizoune, A .Bourazza, M .Elouennass, Brucellosis: A cause of meningitis not to neglect,IDCases ;10:97-9,(2017)

28. CDC, Brucellosis Reference Guide: Exposures, Testing and Prevention. Natl Cent Emerg Zoonotic Infect Dis Dis $: 40,(2017)$

29. G.F.Araj, M.M.Kattar, L.G.Fattouh, K.O Bajakian, S.A.Kobeissi,Evaluation of the PANBIO Brucella Immunoglobulin G (IgG) and IgM Enzyme-Linked Immunosorbent Assays for Diagnosis of Human Brucellosis. Clin Vaccine Immunol ;12:1334-5,(2005)

30. World Health Organization, FAO, editors. Brucellosis in humans and animals. Geneva: WHO; (2006).

31. S-B. Dong, D. Xiao, J-Y. Liu, H-M. Bi, Z-R .Zheng, L-D. Wang, et al, Fluorescence 
polarization assay improves the rapid detection of human brucellosis in China. Infect Dis Poverty ; 10:46,(2021)

32. A.S. Lukambagire, Â.J. Mendes, R.F. Bodenham, J.A .McGiven, N.A .Mkenda, C. Mathew, et al, Performance characteristics and costs of serological tests for brucellosis in a pastoralist community of northern Tanzania. Sci Rep ;11:5480,(2021)

33. Y. Alsayed, F. Monem, Brucellosis laboratory tests in Syria: what are their diagnostic efficacies in different clinical manifestations? J Infect Dev Ctries ;6:495500,(2012)

34. E.E. Bukhari, Pediatric brucellosis: An update review for the new millennium. Saudi Med J ;39:336-41,(2018)

35. M.Yohannes Gemechu, J.Paul Singh Gill. Seroepidemiological survey of human brucellosis in and around Ludhiana, India. Emerg Health Threats J ;4:7361,(2011)

36. Havas KA, Ramishvili M, Navdarashvili A, Hill AE, Tsanava S, Imnadze P, et al. Risk factors associated with human brucellosis in the country of Georgia: a case-control study. Epidemiol Infect ;141:45-53,(2013)

37. W.S .Shell, M.L .Sayed, A.A .Samy, G.M .AlSadek, G.M.M.A. El-Hamid, A.H.M .Ali, Using real-time polymerase chain reaction as an alternative rapid method for enumeration of colony count in live Brucella vaccines. Vet World ;10:610-5,(2017) 